\title{
In situ TEM Mechanical Testing: An Emerging Approach for Characterization of Polycrystalline, Irradiated Alloys
}

\author{
Janelle P. Wharry ${ }^{1}$, Kayla H. Yano ${ }^{1}$, Matthew J. Swenson ${ }^{1}$, and Yaqiao Wu ${ }^{1}$ \\ ${ }^{1 .}$ Materials Science \& Engineering, Boise State University, Boise, Idaho, USA
}

In situ transmission electron microscopic (TEM) mechanical testing techniques enable concurrent TEMresolution imaging/video and mechanical testing of sub-micron-sized electron-transparent specimens. Because nuclear materials are often volume-limited, due to constraints imposed either by radioactivity levels or near-surface ion irradiation damage layers, in situ TEM mechanical testing presents great potential for analyzing these small specimen volumes. But thus far, only a few studies have conducted in situ TEM mechanical tests on irradiated or engineering alloys. Irradiated alloy work [1] focused on single-crystal $\mathrm{Cu}$ that was irradiated after the TEM specimen was fabricated, while the oxide dispersion strengthened (ODS) alloy tested [2] was unirradiated. The objective of the present study, then, is to extend the use of in situ TEM mechanical tests to ODS alloys that have previously been irradiated in bulk form, which is a conventional specimen configuration amongst the nuclear materials community.

The high defect density of an irradiated material poses two competing effects. First, material strength is controlled by the interaction between moving dislocations and material defects, so the reduced defect spacing due to irradiation enables measurement of yield stress from miniaturized samples comparable to that from macroscopic tests. However, when further reducing sample size, the stress required to operate ever-smaller dislocation sources must overcome the strength introduced by the radiation-induced defects. Thus, there may be a threshold sample size below which yield stress from in situ TEM tests over predicts that from bulk measurements. Determining this threshold is critical for analysis.

An Fe-9Cr ODS alloy (Fe-8.67Cr-1.95W-0.28Y-0.23Ti-0.14C-0.048Si-0.06Ni, in wt.\%) is studied here. Irradiations were carried out to 100 displacements per atom (dpa) at $500^{\circ} \mathrm{C}$ with $5 \mathrm{MeV} \mathrm{Fe}^{++}$ions. Both unirradiated and irradiated ODS have defect densities $\sim 5.7 \times 10^{23} \mathrm{~m}^{-3}$, or $\sim 12.1 \mathrm{~nm}$ obstacle spacing calculated from ref. [3]. Focused ion beam was used to machine compression pillars having rectangular cross-sections (following the technique in ref. [4]) with varying minimum dimensions between $75 \mathrm{~nm}$ and $600 \mathrm{~nm}$. Pillars (Fig. 1) were tested using a Hysitron PI95 fitted with a diamond flat punch.

The compressive yield strength is determined using the 0.002 strain offset method from the stress-strain curve measured during each pillar test. Seventeen unirradiated pillars and three irradiated pillars are tested. Macroscopic measurements provide a range of 1000-1200 $\mathrm{MPa}$ [5], [6] for the unirradiated ODS yield stress, and 1100-1600 MPa [5], [6] for the irradiated yield stress. Thirteen of the unirradiated pillars have yield stresses with error bars that fall within or overlap the expected range, while only four have yield stresses greater than the expected range. All three of the irradiated pillars have yield stresses with error bars that fall within or overlap the expected range. Yield stresses are shown as a function of minimum pillar dimension (Fig. 2 left) and pillar volume (Fig. 2 middle). These plots show that there is no apparent size threshold below which pillars deviate from bulk-like yield stress. Ongoing work aims to identify the source of the wide variation in yield stresses measured from small-volume pillars. The elastic modulus is also obtained from each compression test. Measurements on the unirradiated pillars are 20-50 GPa, which is an order of magnitude lower than expected values of 200 GPa from macroscopic tests [7]. This difference arises because the measurement does not account for deformation 
accommodated in the base material below the pillar [4]; future work will develop finite element models to aid in explaining this difference. Irradiated pillars exhibit an increased modulus over the unirradiated pillars, and there is no apparent pillar size effect on elastic modulus (Fig. 2 right).

Results suggest that the high defect density in ODS and irradiated ODS alloys enable quantitative mechanical property measurements from nanosized specimens, without a pillar size effect. This work reinforces that in situ TEM mechanical testing offers tremendous potential for advancement of our understanding of deformation mechanisms in irradiated materials. The talk will also describe additional testing geometries such as microcantilever bend tests and thin foil indentation for irradiated alloys.

\section{REFERENCES}

[1] D. Kiener, et al., Nat. Mater. 10 (2011) p. 608.

[2] B. Girault, et al., Adv. Eng. Mater. 12 (2012) p. 385.

[3] M. J. Swenson and J. P. Wharry, J. Nucl. Mater. 467 (2015) p. 97.

[4] P. J. Imrich, et al., JOM 67 (2015) p. 1704.

[5] C. Robertson, et al., J. Nucl. Mater. 426 (2012) p. 240.

[6] J. Henry, et al., J. Nucl. Mater. 417 (2011) p. 99.

[7] L. Toualbi, et al., J. Nucl. Mater. 442 (2013) p. 410.

[8] We acknowledge Nuclear Regulatory Commission Grant NRC-HQ-84-14-G-0056, US DOE Nuclear Science User Facilities 15-569, the Micron Foundation, and the Center for Advanced Energy Studies.

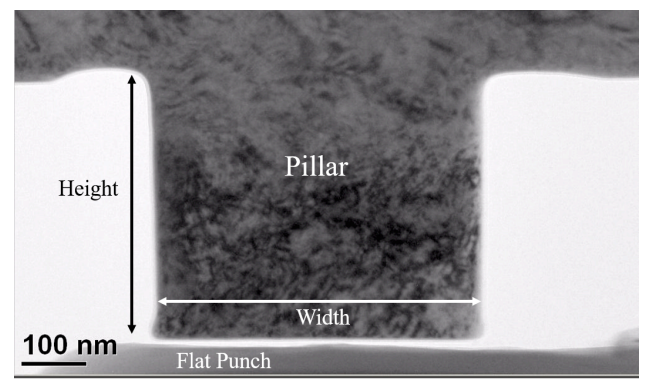

Figure 1. TEM image of a single microcompression pillar, near contact with the flat punch.
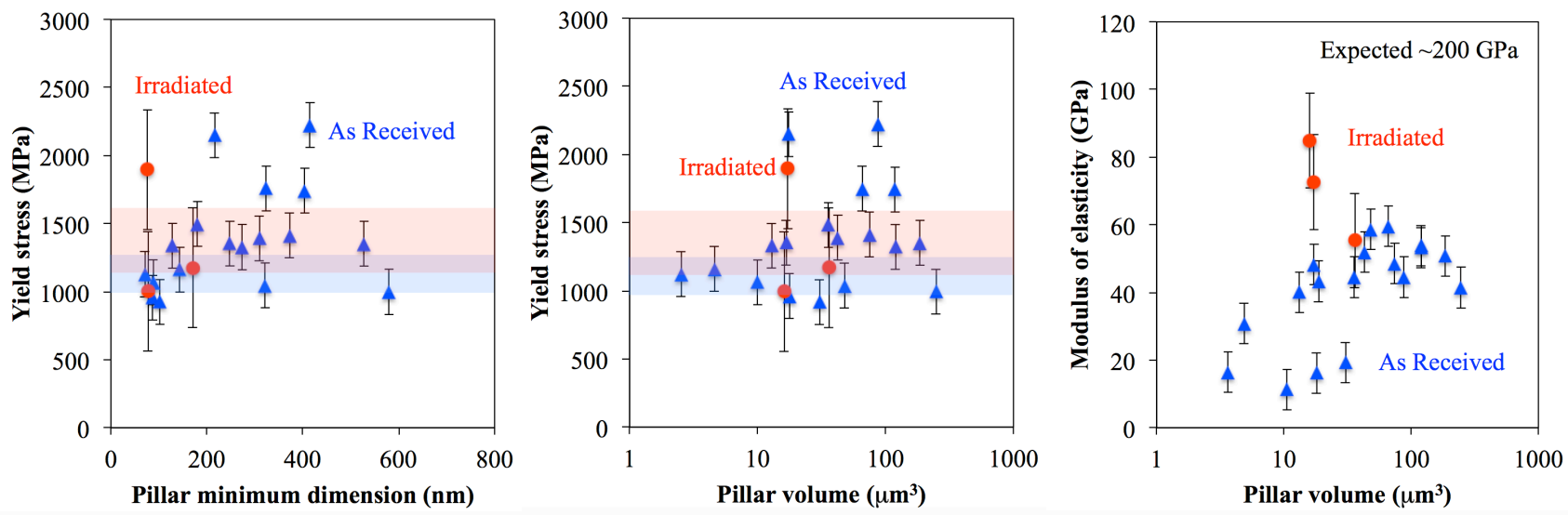

Figure 2. Compressive yield stress $(\mathrm{MPa})$ as a function of (Left) minimum pillar dimension and (Middle) pillar volume. (Right) Modulus of elasticity as a function of pillar volume. Unirradiated pillars shown in blue, irradiated in red, and expected values as blue or red bands, respectively. 